\title{
Femtosecond spectral phase shaping for CARS spectroscopy and imaging
}

Sytse Postma, Alexander C. W. van Rhijn, Jeroen P. Korterik, Jennifer L. Herek, and Herman L. Offerhaus

Optical Sciences Group, Department of Science and Technology, MESA ${ }^{+}$Institute for Nanotechnology, University of Twente,

P.O. Box 217, 7500 AE Enschede, The Netherlands

E-mail: s.postma@utwente.nl

\begin{abstract}
Coherent Anti-Stokes Raman Scattering (CARS) is a third-order non-linear optical process that provides label-free, chemically selective microscopy by probing the internal vibrational structure of molecules. Due to the resonant enhancement of the CARS process, faster imaging is possible compared to Raman microscopy. CARS is unaffected by background fluorescence, but the inherent non-resonant background signal can overwhelm the resonant signal. We demonstrate how simple phase shapes on the pump (and probe) beam reduce the background signal and enhance the resonant signal. We demonstrate chemically selective microscopy using these shaped pulses on plastic beads.
\end{abstract}

\section{Introduction}

Coherent anti-Stokes Raman scattering (CARS) has been used successfully in spectroscopy and microscopy since the development of (tunable) pulsed laser sources. In resonant CARS, molecular vibrations are coherently excited by a pump $\left(\omega_{p}\right)$ and Stokes $\left(\omega_{s}\right)$ pulse. Subsequently a probe $\left(\omega_{p r}\right)$ pulse, which is often similar to the pump pulse, generates the anti-Stokes signal $\left(\omega_{c}=\omega_{p}-\omega_{s}+\omega_{p r}\right)$. The resonant CARS signal is accompanied by an inherent non-resonant background. Here we demonstrate how spectral phase shaping strategies can amplify the resonant features in the spectrum to such an extent that spectroscopy and microscopy can be done at high spectral resolution, even on the integrated spectral response [1]. We use this technique for chemical selective imaging of polystyrene (PS) and polymethylmethacrylate (PMMA)

\section{Setup}

We use a tunable Ti:Sapphire oscillator with a FWHM of $20 \mathrm{~nm}(80 \mathrm{MHz}$ repetition rate). The liquid crystal device (LCD) of the reflective spectral phase shaper has 4096 pixels with a pixel size of $1 \mu \mathrm{m}$ by $6 \mathrm{~mm}$ and a pitch of $1.8 \mu \mathrm{m}$. Effectively the spectral phase shaper has $\sim 600$ degrees of freedom for pattering. For the absolute positioning of phase profiles the complete number of pixels can be used, which implies a positioning precision of $14 \mathrm{GHz}\left(0.5 \mathrm{~cm}^{-1}\right)$. Further details of the spectral shaper setup can be found in an earlier publication [2]. The shaped Ti:Sapphire pulses are used as the pump and probe pulses in the CARS process. The Stokes pulse is generated by a $15 \mathrm{ps}\left(1 \mathrm{~cm}^{-1}\right) \mathrm{Nd}$ :YVO laser. A reflective objective of $0.65 \mathrm{NA}$ is used to focus the light on the sample. The collection objective is a $0.65 \mathrm{NA}$ regular glass objective. The collected light is detected by a spectrometer or a photomultiplier tube. 


\section{Spectroscopy}

Our spectroscopic method is based on sweeping a $\pi$-phase step through the spectrum of the broadband (pump and probe) pulse and recording the CARS spectrum for each position of the step [1]. In this 2D plot the signal from vibrational resonances and the signal from the non-resonant background can be easily identified as distinct features. The difference between the positive and negative $\pi$-phase step rejects purely nonresonant features. Figure 1 shows the spectra for (a) a positive step sweep, (b) a negative step sweep, (c) the difference between (a) and (b) and (d) the integrated CARS signals. The horizontal axes represent the frequency of the phase step and the vertical axes represent the CARS spectra or the integrated signal. The intensities in the figures have been normalized to the unshaped CARS intensity. The strong resonance is clearly identified, the weaker resonances are lost in the noise.
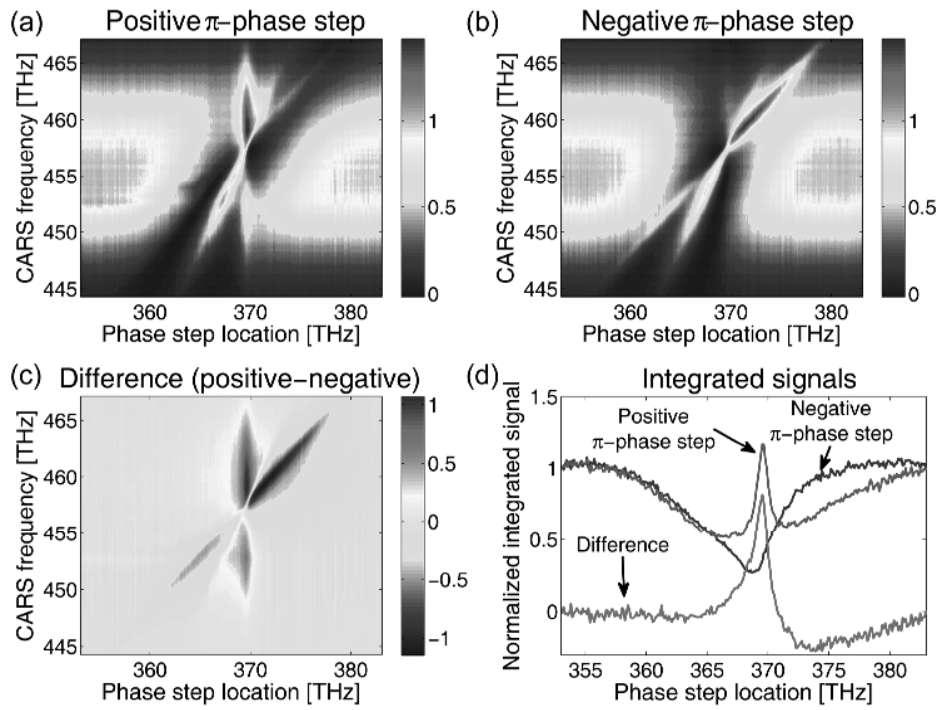

Fig. 1. CARS spectra for a $\pi$-phase step sweep on acetone. a) Positive $\pi$-phase step sweep. b) Negative $\pi$-phase step sweep. c) The difference between the positive and negative sweeps. d) The integrated signal for the three cases.

The imaging of the plastic beads is based on the integrated difference signal. Figure 1(d) shows that, for a slightly red shifted frequency for the phase step in comparison with the location of the vibrational resonance, the difference signal is positive. For blue shifted frequencies the difference signal is negative. In a sample of mixed $4 \mu \mathrm{m}$ PS and PMMA beads dried on a glass substrate, an ordinary transmission image can not resolve the different types of beads. Figure 2 shows integrated difference between the CARS signal for a positive and a negative phase profile. Figure 2(a) shows the CARS image for transform limited pulses (flat phase profile). Figure 2(b-d) show difference CARS images, with (b) the difference of two $\pi$-phase steps at $372.7 \mathrm{THz}$, (c) the difference of two $\pi$-phase steps at $369.0 \mathrm{THz}$, and (d) the difference of two phase structures with two phase steps. The images have been normalized by the maximum CARS signal for the transform limited pulse. 
(a) Transform limited

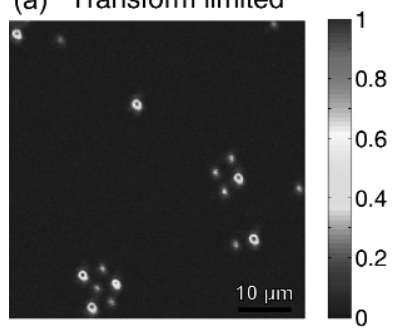

(c) PMMA profile

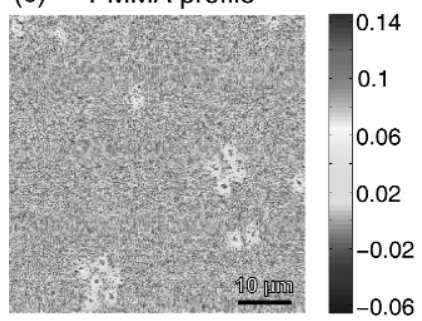

(b) Polystyrene profile

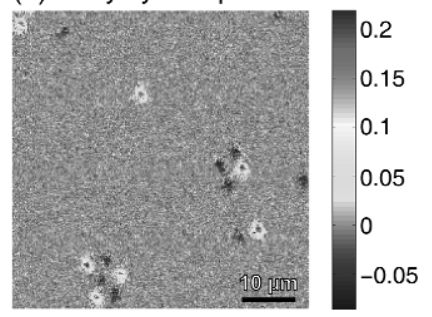

(d) Double step

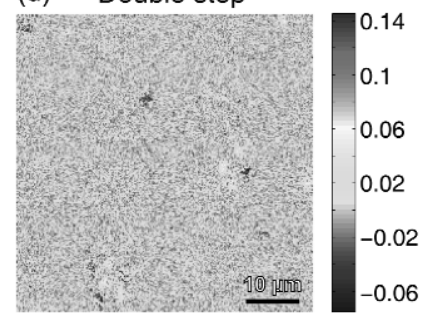

Fig. 2. Spectral phase profiles applied to the pump and probe pulses, which result in chemical contrast as a result of the applied phase profile. a) flat phase profile. b) PS enhanced phase profile. c) PMMA enhanced phase profile. d) PMMA enhanced and PS decreased phase profile.

In this particular case the transform limited CARS image also shows a difference between the PS and PMMA beads, because PS has a larger cross-section than PMMA for the chosen spectra of the pump and probe and Stokes. Figure 2(b) shows a CARS image for an applied phase profile that enhances the resonant signal of the PS beads and it results in a net negative result for the PMMA beads. Figure 2(c) shows a CARS image for an applied phase profile that enhances the resonant signal of the PMMA beads, which results in a similar net result for the PS beads. Figure 2(d) shows the same image for an applied phase profile that enhances the PMMA beads and at the same time results in a net negative result for the PS beads.

The subtraction of opposite phase profiles results in a removal of all pure nonresonant contributions. The concurrent phase step in the probe pulse results in less (resonant) CARS signal, which reduces the signal to noise ratio. The low signal to noise ratio is especially visible in the case for the double step image.

\section{Conclusions}

We demonstrate a method for chemically selective imaging based on detection of the integrated CARS signal, by applying simple phase profiles to the pump and probe pulses of the CARS process. For the future we are planning to expand these techniques for use in the fingerprint region for biological purposes.

[1] S. Postma, A. C. W. van Rhijn, J. P. Korterik, P. Gross, J. L. Herek, and H. L. Offerhaus, Opt. Express 16, 7985 (2008).

[2] S. Postma, P. van der Walle, H. L. Offerhaus, and N.F. van Hulst, Rev. Sci. Instrum. 76, 123105 (2005). 